\title{
CORRIENTES ELÉCTRICAS ALINEADAS CON EL CAMPO GEOMAGNÉTICO EN BAJAS LATITUDES MAGNÉTICAS
}

\author{
Joel Rojas Acuña1 y Jorge A. Bravo Cabrejos2
}

$* *$ Naoshi Fukushima.

SUMILLA: La existencia de las corrientes eléctricas alineadas con el campo geomagnético en bajas latitudes magnéticas del Perú ha sido inferida utilizando los métodos de Gettemy[1] y Fukushima[2] aplicados a los datos magnéticos registrados durante el Año Geofísico Internacional (AGI) y el Año Internacional del Electrochorro Ecuatorial (AIEE) en el Perú. La representación de la variación magnética diaria quieta solar, Sq, usando mapas de contorno magnéticos de las componentes $\mathrm{D}, \mathrm{H}$ y Z del campo geomagnético registrado en el Observatorio Magnético de Huancayo (OMH) para los dos periodos (1957-1958 y 1990-1991) permite visualizar los cambios estacionales en la variación diaria. Los resultados obtenidos son similares a los obtenidos por Gettemy para el Observatorio Magnético de Koror (OMK) en las Islas Palau (Océano Pacifico) durante el AGI. Los mapas de contorno magnéticos para las componentes D, $\mathrm{H}$ y Z del campo geomagnético registrado en el Observatorio Magnético de Ancón (OMA) para el periodo de 1990-1991 son también muy similares a los de Huancayo y Koror, aún en épocas diferentes. Asimismo, los mapas de contorno magnéticos para otras cinco estaciones magnéticas cerca al Ecuador magnético (1958) han sido elaborados para fines de comparación e interpretación. Dos diagramas, referidos a los patrones del sistema de corrientes eléctricas superiores de la variación magnética diaria quieta solar, Sq, para 1/2(verano + invierno) y 1/2(verano - invierno) en bajas latitudes magnéticas del Perú, fueron elaborados utilizando el método de análisis de las variaciones magnéticas diarias en tierra propuesto por Fukushima [2] para medianas latitudes geomagnéticas aplicado a cuatro estaciones magnéticas del Japón. Los resultados obtenidos nos permiten inferir las corrientes eléctricas alineadas con el campo geomagnético en bajas latitudes y confirmar la inversión estacional en la variación magnética diaria de la declinación en el OMH y en el OMA.

ABSTRACT: The existence of electric currents aligned with the magnetic field at low latitudes has been inferred using the methods of Gettemy [1] and Fukushima [2] applied to the magnetic data recorded in Peru during the International Geophysical Year (IGY) and the International Equatorial Electrojet Year (IEEY). The representation of the solar quiet daily magnetic variation, Sq, using magnetic contour maps of the $\mathrm{D}, \mathrm{H}$, and $\mathrm{Z}$ components of the magnetic field recorded at the Huancayo Magnetic Observatory (OMH) for the two time periods(1957 - 1958 and 1990 - 1991) was designed to visualize the seasonal changes in the daily variation. The maps obtained in this work are similar to the ones obtained by Gettemy [1] for the Magnetic Observatory at Koror (OMK) at the Palao Islands (Pacific Ocean) during IGY. The magnetic contour maps for the components of the magnetic field recorded at the Observatory of Ancon (OMA) for the period of 1990 - 1991are also very similar to the ones from Huancayo and Koror, even in different time periods. Likewise, the magnetic contour maps for 5 other magnetic stations at other equatorial sites (1958) are prepared for the purpose of comparison and interpretation. Two diagrams referred to the patterns of the upper electric current system of the solar quiet magnetic variation, Sq, for 1/2 (Summer + Winter) and 1/2(Summer - Winter) at low magnetic latitudes were prepared using the method of analysis for the ground daily magnetic variations as proposed by Fukushima [2] for middle magnetic latitudes and applied to four magnetic magnetic stations in Japan. The results obtained allow us to infer the aligned electric currents with the geomagnetic field at low latitudes and confirm the seasonal inversion in the daily magnetic variation of the declination at the $\mathrm{OMH}$ and in the OMA.

\section{INTRODUCCIÓN}

Se ha propuesto que las corrientes eléctricas alineadas con el campo geomagnético (CAC) en la magnetósfera fluyen no sólo en altas latitudes sino también en medianas y bajas latitudes magnéticas [2,3,4,5]. Sin embargo, su detección con satélites científicos sólo ha sido posible en la región de altas latitudes magnéticas. El satélite científico 1963-38C detectó las CAC en altas latitudes magnéticas [6]. Fukushima [2] ha propuesto que la existencia de estas CAC en medianas y bajas latitudes magnéticas se puede inferir de un análisis de los magnetogramas registrados en tierra, en particular, a partir de la diferencia estacional en la variación diurna del campo geomagnético. A medianas latitudes magnéticas, la componente dominante de las CAC son las corrientes eléctricas transecuatoriales del hemisferio de invierno al de verano que combinan las regiones de focos de corrientes eléctricas asociados a la variación magnética diaria quieta solar, Sq, en los hemisferios norte y sur. Las CAC en medianas latitudes magnéticas han sido discutidas por van Sabben [4,5,7], Yanagihara [8], Maeda [9], Mishin [29], Fukushima [2,3,10] y Wagner [11]. Fukushima [2] fue el primero en inferir las CAC en medianas latitudes geomagnéticas, con dirección del hemisferio en invierno al de verano, sobre la base de los datos observados en tierra en cuatro estaciones magnéticas en Japón en 1989. Según Fukushima [3] existen dos sistemas de CAC en bajas latitudes magnéticas (cerca del ecuador magnético). La dirección de estas CAC es del hemisferio en verano al hemisferio en invierno en las horas anteriores al medio día y del hemisferio en invierno al hemisferio en verano en las horas de la tarde, según la dependencia estacional de la variación magnética diaria quieta solar, Sq, de un observatorio temporal (Koror) observada en los mapas de contorno magnéticos, según Gettemy [1] y Fukushima, [2]. Hasta el momento, estas CAC en bajas latitudes magnéticas no han podido ser detectadas desde satélites y se ha propuesto como hipótesis de este trabajo que, de existir éstas y de tener suficiente intensidad, pueden producir variaciones características en los registros magnéticos que se toman en tierra. Adicionalmente, se plantea que la intensidad y direccionalidad de estas CAC dependen tanto de la iluminacion diferencial de los hemisferios del norte y sur durante las estaciones del año asi como de la hora local media en cada estación. Teniendo como base esta hipótesis de trabajo y la metodología de análisis de los datos magnéticos desarrollados por otros investigadores [1,2], se ha procesado los datos magnéticos tomados en estaciones permanentes y temporales, ubicadas en Perú, para los periodos del AGI (1957-1958) y del AIEE (1990-1991). Previamente, para fines de comparación y comprobación de los programas de cálculo desarrollados, se ha aplicado esta metodología a datos de estaciones magnéticas ubicadas en otras partes del mundo que han sido procesados por otros investigadores, obteniéndose resultados 
satisfactorios. También se ha aplicado este análisis a otras estaciones magnéticas ecuatoriales del mundo para fines de comparación y verificación de la consistencia de la hipótesis de trabajo.

El objetivo es inferir las corrientes eléctricas alineadas con el campo geomagnético en bajas latitudes magnéticas según su efecto magnético registrado en tierra e identificar el cambio estacional en las componentes del campo geomagnético en la superficie de la Tierra, generado por la iluminación diferencial de los hemisferios norte y sur durante el año. En la sección II se menciona los antecedentes teóricos que apoyan la teoría de las CAC inter - hemisféricas en bajas, medianas y altas latitudes magnéticas. En la sección III se describe los datos usados para obtener los mapas de contorno para Huancayo y Ancón y otras partes del globo terrestre (Koror, Jarvis, Trivandrum y Addis Ababa), y el patrón de corrientes eléctricas superiores en la alta atmósfera (arriba de la superficie de la Tierra) para una red de cinco estaciones magnéticas ecuatoriales peruanas (Talara, Chiclayo, Chimbote, Huancayo y Yauca). En la sección IV se presenta la discusión de los resultados obtenidos y las conclusiones. Los mapas de contorno magnéticos dan buena información del cambio estacional en la variación diaria de las componentes D, H y Z del campo geomagnético en el Observatorio Magnético de Huancayo (OMH) durante el AGI y en el Observatorio Magnético de Ancón (OMA) durante AIEE. Similarmente, se ha elaborado los mapas de contorno para cinco observatorios magnéticos ecuatoriales como son Koror, Jarvis, Trivandrum, Addis Ababa y Huancayo durante el AGI (1958) para días quietos. Se observa una inversion estacional de DD en los mapas de contorno magnéticos para el OMA, el OMH, Jarvis, Addis Ababa y en forma no muy clara en Trivandrum. Se observa una débil huella de las CAC en los diagramas de patrones de corrientes eléctricas superiores en la mañana y en la tarde en Peru.

\section{FUNDAMENTOS TEÓRICOS}

\subsection{Corrientes eléctricas externas e internas a la Tierra y su influencia sobre $\mathrm{D}, \mathrm{H}$ y $\mathrm{Z}$ del campo geomagnético}

La variación diaria del campo geomagnético observada en la superficie de la Tierra es causada por dos clases de corrientes eléctricas, una que fluye dentro y por encima de la ionósfera, y la otra que fluye debajo de la superficie en el interior de la Tierra [2]. Estas últimas, que fluyen debajo de la superficie de la Tierra, son aquellas inducidas en el interior de la Tierra debido a la variación temporal del campo magnético producido por las corrientes eléctricas primarias en la ionósfera y la magnetósfera [12]. La inducción electromagnética en el interior de la Tierra depende de la razón de cambio del campo magnético producido por fuentes externas. En el caso extremo, para una onda de radio ordinaria de alta frecuencia, la superficie de la Tierra se comporta de modo semejante a un conductor perfecto. Por el efecto de escudamiento de las corrientes eléctricas que fluyen en la superficie de la Tierra, la intensidad del campo magnético observado sobre la superficie de la Tierra se duplica para las componentes $\mathrm{H}$ y D (paralelas a la superficie de la Tierra), mientras que la componente $\mathrm{Z}$ (perpendicular a la superficie de la Tierra) tiende a desaparecer completamente. Ya que los rangos de variación del campo magnético observados en la práctica son del orden de 10-1 nT/s (para tiempos perturbados) a 10-3 $\mathrm{nT} / \mathrm{s}$ (para tiempo quieto), la Tierra se comporta como un conductor imperfecto con propiedades eléctricas y magnéticas que dependen de la topografía de la superficie de la Tierra y la distribución de la materia altamente conductora y altamente permeable dentro de la Tierra [2]. Bajo estas circunstancias la variación diaria de DZ observada en un observatorio terrestre es influenciada en gran medida por la topografía y la estructura subterránea en la vecindad del observatorio de interés (como se verá después, por ejemplo, en algunos observatorios magnéticos ubicados en islas). Por otro lado, en las variaciones diarias de D y H observadas sobre la Tierra, la contribución de las corrientes eléctricas externas primarias es usualmente amplificada (por aproximadamente 1.5 veces) por las corrientes eléctricas inducidas dentro de la Tierra, porque el campo magnético producido por estas últimas corrientes eléctricas inducidas está casi en la misma dirección como aquél de las corrientes eléctricas externas. Esta es la razón por qué los geomagnetístas consideran un sistema de corrientes eléctricas superiores incluyendo sólo las variaciones en las componentes $\mathrm{H}$ y D observadas del campo geomagnético, mientras que la variación de la componente $\mathrm{Z}$ es más relevante en el sondeo electromagnético de la estructura subterránea [12].

\subsection{Efecto magnético en tierra producido por corrientes eléctricas alineadas con el campo geomagnético.}

Cuando el efecto magnético en tierra de las CAC es calculado con la ayuda de la ley de Biot-Savart, debemos tratar siempre con un circuito de corriente eléctrica cerrado en el espacio sobre la Tierra. En altas latitudes magnéticas, el campo magnético producido por una corriente eléctrica alineada que ingresa casi verticalmente sobre la zona auroral (que produce un campo con sentido horario visto desde arriba de la Tierra) es aproximadamente cancelado por una corriente eléctrica que se esparce horizontalmente en la ionósfera desde el punto de ingreso de la corriente eléctrica vertical proveniente de la magnetósfera (ver figura 2 en [3]). La cancelación es perfecta si la corriente eléctrica desde la magnetósfera es exactamente vertical y la ionósfera tiene una conductividad de Pedersen uniforme y ninguna conductividad de Hall. Por lo tanto, el campo magnético observado en tierra y producido por las CAC en la magnetósfera, que está enlazado a corrientes eléctricas horizontales en la ionósfera, depende de: (1) una incidencia oblicua de las CAC en la ionósfera; (2) no uniformidad de la conductividad de Pedersen en la ionósfera, y (3) la existencia de la conductividad de Hall en la ionósfera [13,14,15]. Las CAC en medianas latitudes magnéticas han sido discutidas por van Sabben [4,5,7], Maeda [9], Fukushima [2,10] y otros. La cantidad total de CAC entre los hemisferios norte y sur en el lado iluminado por el Sol, según Fukushima [2], ha sido estimada ser del orden de $105 \mathrm{~A}$. Si se supone que esta corriente eléctrica fluye sólo en un área de $20^{\circ} \times 20^{\circ}$ alrededor del foco de corriente eléctrica Sq, la densidad de corriente eléctrica en el nivel ionosférico es mucho menor que $1 \mathrm{~mA} / \mathrm{m} 2$, y es difícil detectar una corriente eléctrica tan débil en el espacio.

\subsection{Corrientes eléctricas alineadas con el campo geomagnético en diferentes latitudes}

La distribución real de las corrientes eléctricas en el espacio que rodea a la Tierra es tridimensional, consistiendo de corrientes eléctricas horizontales que fluyen en la ionósfera (gas cuya dinámica es gobernada por colisiones entre las partículas cargadas y neutras) y CAC en la magnetósfera (medio con ausencia de colisiones). Aunque no hay conversión única de un sistema de corrientes eléctricas equivalentes bidimensional a uno tridimensional, es completamente natural pensar de alguna CAC transecuatorial que fácilmente fluiría aún bajo un pequeño diferencial en los valores del potencial eléctrico en la ionósfera entre un par de puntos conjugados en los hemisferios norte y sur (la conductividad eléctrica en la dirección N-S a 250 km de altitud es 103 veces que en el nivel de $100 \mathrm{~km}$ ) [2]. El desarrollo de satélites científicos con magnetómetros de tres ejes hizo posible detectar las 
CAC en altas latitudes magnéticas, primero por el satélite 1963-38C [6] según el gradiente espacial muy inclinado de la persistente componente este - oeste del campo geomagnético detectado encima de las zonas aurorales norte y sur. Estudios posteriores con los satélites científicos TRIAD, MAGSAT, HILAT y algunos otros satélites, permitieron revelar varias importantes características de las CAC en altas latitudes magnéticas, incluyendo su persistencia y sistemática dependencia de su dirección (tanto en su entrada como en su salida) de la hora local, latitud magnética, y tanto como de la condición del viento solar. Aunque las CAC en la magnetósfera han sido detectadas sólo por encima de las zonas aurorales en altas latitudes magnéticas, es natural pensar de la presencia de CAC encima de la ionósfera también, en medianas y bajas latitudes magnéticas, porque ellas tienden a fluir cuando se presenta una pequeña diferencia de potencial eléctrico en la ionósfera entre un par de estaciones conjugadas en los hemisferios norte y sur, aunque la densidad de corriente eléctrica no sea lo suficientemente intensa como para ser detectadas por intrumentos geomagnéticos ubicados en los satélites.

\subsubsection{Corrientes eléctricas alineadas con el campo geomagnético en medianas latitudes magnéticas}

En el centro del vórtice de corriente eléctrica Sq, el potencial eléctrico en la región dínamo muestra un valor mínimo en ambos hemisferios, tanto en el hemisferio de verano como en el hemisferio de invierno. Por lo tanto, una CAC fluirá desde el hemisferio de invierno al hemisferio de verano, porque el valor del potencial eléctrico es menor en el de verano. Tales CAC desde/hasta el foco de la corriente eléctrica Sq fue abogado por van Sabben [5,7,16], Maeda [9] y otros. La figura 2 en Fukushima [2] muestra un diagrama esquemático para el efecto magnético en tierra de una CAC conectada a las corrientes eléctricas de retorno que se esparce sobre toda la ionósfera. El efecto magnético resultante en tierra es dado por su corriente eléctrica equivalente (por líneas a trazos) y la corriente eléctrica de retorno que diverge en la ionósfera desde el punto $\mathrm{N}$ es remplazado simplemente por una corriente eléctrica de línea hacia arriba. En general, el efecto magnético neto en tierra de una CAC se anula hasta una considerable extensión (dependiendo también del ángulo magnético observado o dip), por el efecto de su corriente eléctrica de retorno que fluye en la ionósfera. Fukushima [2] demostró la existencia de las CAC transecuatoriales a partir de la dependencia estacional de las variaciones magnéticas Sq en medianas latitudes magnéticas. Estas corrientes eléctricas fluyen sin interactuar con el electrochorro ecuatorial. Fukushima usó los valores horarios de 4 observatorios magnéticos (Memambetsu, latitud geomagnética 34,6; Kakioka, 26,8; Kanoya, 21,1² Chichijima, 17,8) correspondientes al año 1989; calculó las variaciones geomagnéticas diarias medias de DX, DY y DZ para 5 días quietos en los meses de verano (Mayo, Junio, Julio y Agosto) e invierno (Enero, Febrero, Noviembre y Diciembre) del hemisferio norte. Luego, los resultados son mostrados para 1/2 (Verano + Invierno) en la figura 1 y $1 / 2$ (Verano - Invierno) en la figura 2 en Fukushima [2]. Donde DX y DY son dados para indicar la dirección de las corrientes eléctricas superiores. Fukushima [2] mostró que el sistema de corrientes eléctricas Sq promedio revela un vórtice de corriente eléctrica antihorario en medianas latitudes magnéticas en el hemisferio norte y que su centro está localizado entre las 11 y 12 horas tiempo local, asociado con un valor DZ negativo (hacia arriba) y grande. El sistema de corrientes eléctricas Sq es atribuido principalmente a la corriente eléctrica de Hall en la ionósfera inferior alrededor de la región de potencial eléctrico negativo. La corriente eléctrica Sq en el hemisferio sur fluye en sentido horario, visto desde arriba de la Tierra, produciendo valores DZ positivos (hacia abajo). Esto significa que el potencial eléctrico cerca al centro del vórtice Sq en el hemisferio sur es también negativo como en el hemisferio norte. Si el patrón de corriente eléctrica Sq e intensidad son simétricos con respecto al ecuador, no habría diferencia de potencial eléctrico entre los hemisferios norte y sur para producir las CAC interhemisféricas. Las CAC fluirán a través de la magnetósfera para cancelar o al menos reducir la diferencia de potencial entre los lugares conjugados norte y sur, cuando tal diferencia de potencial comience a tomar lugar. Si las corrientes alineadas transecuatoriales con el campo fluyen hacia el hemisferio norte, su incidencia oblicua en la ionósfera produce un efecto magnético en tierra que puede ser expresado por un sistema de corrientes eléctricas superior equivalente a un par de lazos de corriente eléctrica circular (un lazo de corriente eléctrica con sentido horario con un DZ negativo sobre el lado oeste de la región de incidencia de las CAC en el hemisferio norte). Al observar la figura 2 en Fukushima [2], notamos un área con DZ negativo resaltante a las 9 hs TL sobre Kakioka, Kanoya y Chichijima asociado con un sentido de corriente eléctrica superior antihorario. Esto puede ser considerado una huella en tierra de las CAC en medianas latitudes geomagnéticas que fluyen de 11 a 12 hs TL en la ionósfera oblícuamente (ver figura 2 en Fukushima[3], aunque sólo podemos ver una débil huella del lazo de corriente eléctrica horario sobre el lado de la tarde del hemisferio iluminado por el Sol [2]. Según Fukushima [12] la razón por la cual las corrientes eléctricas antihorarias en las mañanas son más resaltantes que las corrientes horarias en la tarde parece originarse de un gradiente espacial más inclinado de la conductividad ionosférica.

\subsubsection{Corrientes eléctricas alineadas con el campo geomagnético en bajas latitudes magnéticas en la zona ecuatorial del Océano Pacífico.}

Una característica muy interesante de la variación magnética diaria quieta solar, Sq, en un observatorio temporal AGI en Koror (7 $20^{\prime} \mathrm{N}, 134^{\circ} 30^{\prime} \mathrm{E}$ ) fue reportado por Gettemy [1]. La estación estuvo justo sobre el ecuador magnético; el valor medio mensual de Z fue $0 \mathrm{nT}$ en Julio de 1957 y -84 nT en Diciembre de 1958. La figura 3 en Fukushima [3] muestra la variación magnética diaria en Koror para días quietos para las tres componentes del campo geomagnético, donde observamos que las variaciones diarias DD están invertidas en las estaciones del hemisferio de verano e invierno. La dependencia estacional de DD fue reportada por muchos investigadores [17,18,19,20]. Aunque el campo magnético en tierra de las CAC es considerablemente reducido en medianas latitudes magnéticas por las corrientes eléctricas de retorno que fluyen en la ionósfera, el efecto es perceptible en la región ecuatorial para CAC en bajas latitudes magnéticas a causa de un pequeño ángulo de inclinación observado o dip. DZ es siempre positiva en la mañana y negativa en la tarde a través de todo el año. Esta tendencia es razonablemente interpretada por la corriente eléctrica de inducción en el mar causada por el desarrollo y decaimiento diario del electrochorro ecuatorial (que fluye con sentido hacia el este) sobre la estación de Koror, la cual está situada sobre el lado sur-este de las Islas de Palau [2]. Se puede inferir la existencia de CAC en bajas latitudes magnéticas según un análisis de los datos magnéticos en tierra, en particular de la dependencia estacional de la variación diurna en la declinación magnética. Esta inversión estacional es atribuida a las corrientes eléctricas equivalentes transecuatoriales del campo Sq a CAC inter-hemisféricas en la magnetósfera en bajas latitudes magnéticas. La inversión estacional de la variación diaria de DD en la región ecuatorial es atribuida a un par de CAC en el hemisferio iluminado por el Sol cerca a los meridianos de amanecer y anochecer, como se ilustra esquemáticamente en la figura 1 de Fukushima [3]. En comparación con las CAC en altas latitudes magnéticas, la densidad de corriente en latitudes medianas y bajas es muy pequeña (mucho menos que $1 \mathrm{~mA} / \mathrm{m} 2$ ) para ser detectada por instrumentos a bordo de satélites, pero esto no implica del todo la ausencia de 
CAC allí. Es también útil mencionar que el efecto no deseable de las propiedades eléctricas y magnéticas del subsuelo vecino al sitio de cada observatorio es casi eliminado en los diagramas calculados para 1/2 (verano-invierno) en figura 2 en Fukushima [2]; así que estos diagramas son útiles para ver el efecto de CAC en medianas y bajas latitudes magnéticas sin las serias influencias no deseables de las corrientes eléctricas de inducción debajo de la tierra.

\subsection{Corrientes eléctricas alineadas con el campo geomagnético causadas por la asimetría norte-sur del campo magnético ecuatorial Sq}

Si interpretamos la corriente eléctrica equivalente del campo Sq, que cruza el Ecuador, como CAC interhemisféricas en la magnetósfera en bajas latitudes magnéticas, la inversión estacional de la variación diaria de DD en la región ecuatorial es atribuida a un par de CAC en el hemisferio iluminado por el Sol cerca a los meridianos del amanecer y anochecer, como se muestra esquemáticamente en la figura 1 en Fukushima [3]. Este cuadro muestra también las CAC de invierno a verano entre los focos de corriente eléctrica Sq en los dos hemisferios sobre el lado iluminado por el Sol, la existencia de la cual es vista en la figura 2 en Fukushima [2], para la asimetría verano - invierno del campo Sq en medianas latitudes geomagnéticas en Japón. La intensidad total de estas CAC interhemisféricas en la magnetósfera ha sido estimada ser de unas pocas decenas de kA. El par de CAC cerca a los meridianos del amanecer y anochecer respectivamnte fue propuesto por Schlapp y colaboradores en 1988 [2] después de su comparación de la variación magnética diaria de Sq observada en dos estaciones en medianas latitudes magnéticas en los hemisferios norte y sur. Maeda y Murata [21] indicaron primero la posibilidad de la existencia de un par de CAC en la mañana (en el sentido de verano a invierno) y en la tarde (de invierno a verano) asociadas con la corriente eléctrica Sq en la estación del solsticio. Ellos discutieron la acción de dínamo en la ionósfera para producir corrientes eléctricas Sq por la conductividad ionosférica que fue asumida ser la conductividad de Pedersen. Estudios posteriores de esta clase de problema, en la cual la ionósfera tiene las conductividades de Pedersen y Hall, o sea, aquellos por van Sabben [7] y Fukushima [10], mostraron que la corriente Sq es principalmente la corriente eléctrica de Hall en la ionósfera, con un gran potencial eléctrico negativo en el foco del vórtice de corriente eléctrica Sq y el flujo de CAC en medianas latitudes magnéticas sobre el lado del día es del hemisferio de invierno hacia el hemisferio de verano, a causa de un potencial eléctrico negativo más intenso en el hemisferio de verano.

\section{DATOS}

Los datos magnéticos del OMH durante el AGI (1957-1958) se encuentran publicados en Giesecke y colaboradores [22]. Los datos magnéticos del OMH y del OMA durante el AIEE (1990-1991) fueron obtenidos de Ishitsuka [23]. Los datos magnéticos de días quietos del AGI (1958) de Huancayo, Koror, Jarvis, Trivandrum y Addis Ababa están publicados en Price y Stone [24]. La ubicación de estas estaciones magnéticas se muestran en las figuras 1 y 2. Las ubicaciones de los observatorios cerca al ecuador magnético durante el periodo AGI y el periodo AIEE se muestran en la tabla 1. Los datos usados para obtener los diagramas de patrones del sistema de corrientes eléctricas superiores son los valores medios horarios de las variaciones diarias del campo geomagnético generados por el sistema de corrientes eléctricas que fluyen en la ionósfera, registrados en cuatro estaciones magnéticas temporales (Talara, Chiclayo, Chimbote y Yauca) y un observatorio magnético permanente (Huancayo) durante el periodo 1958-1959 que se encuentran publicados en Forbush y Casaverde [25]. Se utilizó una PC 486 DX del Laboratorio de Cálculo Científico de la Unidad de Post Grado de la Facultad de Ciencias Físicas de la UNMSM para la elaboración y ejecución de todos los programas Fortran. El paquete de programas SURFER fue utilizado para obtener los mapas de contorno magnéticos.

\begin{tabular}{||c|c|c|c|c||}
\hline \multicolumn{5}{|c|}{ Tabla 1 - Estaciones geomagnéticas AGI y AIEE[23,24] } \\
\hline \hline ESTACIÓN & $\begin{array}{c}\text { LATITUD } \\
\text { GEOGRÁFICA }\end{array}$ & $\begin{array}{c}\text { LONGITUD } \\
\text { GEOGRÁFICA }\end{array}$ & IobsAGI & $\begin{array}{c}\text { Iobs } \\
\text { AIEE }\end{array}$ \\
\hline \hline Addis Ababa & $09^{\circ} 02,0^{\prime} \mathrm{N}$ & $38^{\circ} 46,0^{\prime} \mathrm{E}$ & $-0,5^{\circ}$ & \\
\hline \hline Trivandrum & $08^{\circ} 29,0^{\prime} \mathrm{N}$ & $76^{\circ} 57,0^{\prime} \mathrm{E}$ & $-0,3^{\circ}$ & \\
\hline \hline Koror & $07^{\circ} 20,0^{\prime} \mathrm{N}$ & $134^{\circ} 30,0^{\prime} \mathrm{E}$ & $0,0^{\circ}$ & \\
\hline \hline Jarvis Island & $00^{\circ} 23,0^{\prime} \mathrm{S}$ & $160^{\circ} 02,0^{\prime} \mathrm{O}$ & $1,1^{\circ}$ & \\
\hline \hline Huancayo & $12^{\circ} 02,3^{\prime} \mathrm{S}$ & $75^{\circ} 19,4^{\prime} \mathrm{O}$ & $1,0^{\circ} 1,6^{\circ}$ & \\
\hline \hline Ancón & $11^{\circ} 46,4^{\prime} \mathrm{S}$ & $77^{\circ} 08,9^{\prime} \mathrm{O}$ & $1,3^{\circ}$ & \\
\hline \hline Nota: Iobs es el ángulo de inclinación magnética observada \\
\hline \hline
\end{tabular}

Figura 1 - El mapa mundo que muestra las posiciones de las estaciones incluidas en el análisis (Forbes, 1981).

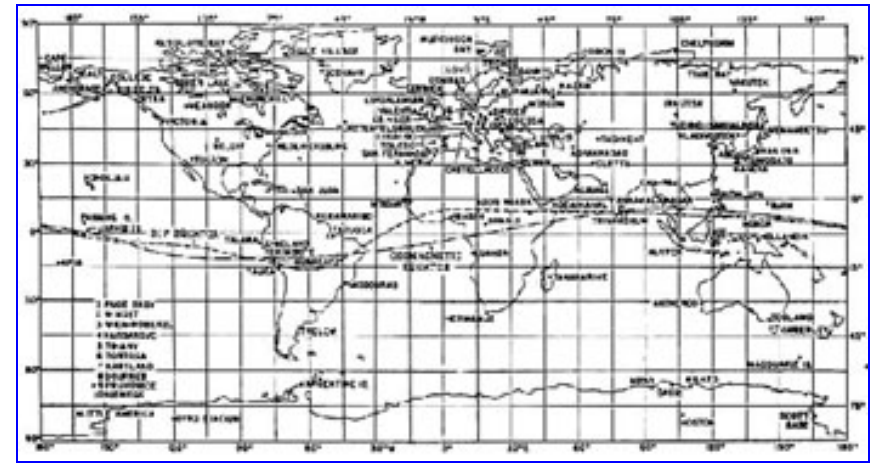


Figura 2 - Observatorios magnéticos permanentes y temporales, usados en el presente trabajo, mostradas en relación al ecuador magnético medio y líneas de igual inclinación calculadas (según Burrows, 1970)

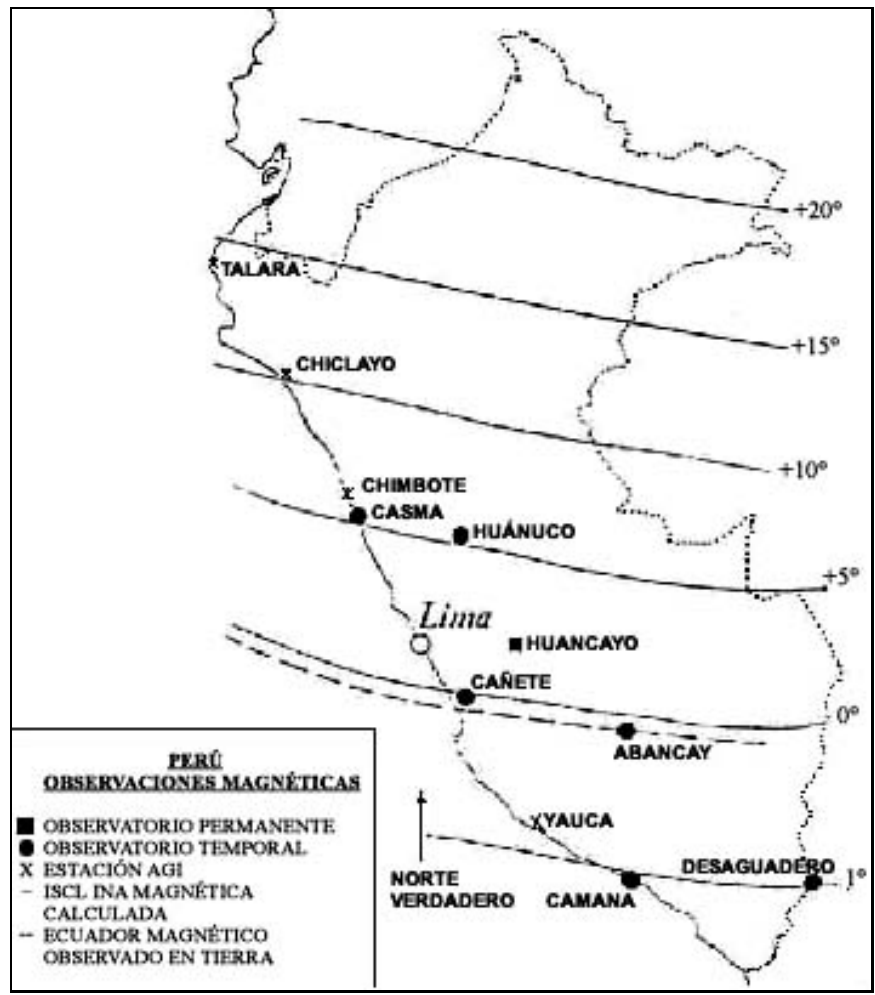

Figura 3 - Variación magnética diaria (desviaciones de los valores medios diarios) para las tres componentes del campo geomagnético sobre dias quietos en Huancayo durante el AGI.

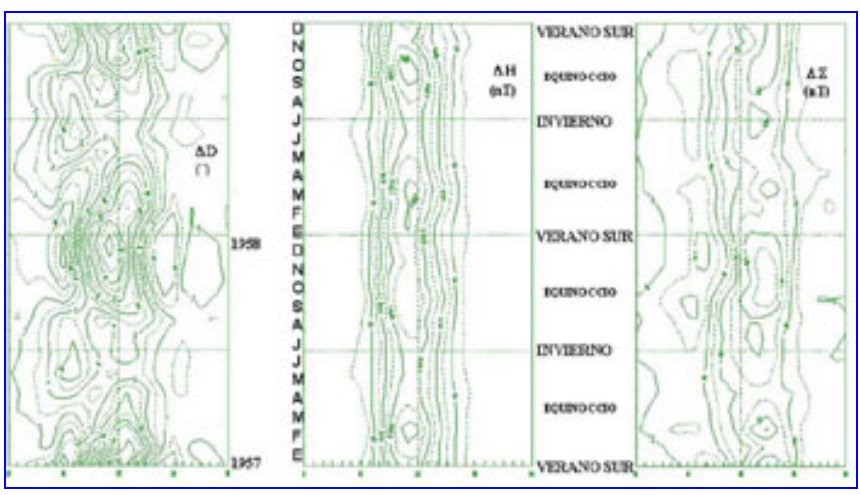




\section{RESULTADOS Y DISCUSIÓN}

\subsection{Variación magnética diaria en Huancayo durante el AGI.}

Cada día el campo magnético en tierra muestra variaciones regulares y/o irregulares, las cuales son atribuidas en parte a corrientes eléctricas que fluyen en el espacio que rodea a la Tierra dentro y por encima de la ionósfera, y parcialmente también a corrientes eléctricas inducidas bajo la superficie de la Tierra debido a la variación temporal del campo magnético externo [2]. En el OMH estas variaciones magnéticas son registradas como trazos contínuos de las componentes $\mathrm{D}, \mathrm{H}$ y Z del campo geomagnético en magnetogramas sobre un variógrafo [25]. El OMH registra estas variaciones desde 1922 en forma contínua y tomó parte muy activa durante el AGI. La ubicación del OMH durante el periodo del AGI se muestra en la tabla 1. Su ubicación sobre el nivel del mar es de 3313 m, se encuentra muy cerca al ecuador magnético observado o comunmente llamado "dip equator", y los datos registrados nos pueden dar información acerca de la naturaleza del electrochorro ecuatorial (EE). Por lo tanto, el carácter de la variación magnética diaria es de especial interés. Se tiene como objetivo estudiar la variación geomagnética diaria en Huancayo cerca al ecuador magnético durante el AGI (1957-58) para compararlo con los trabajos de Gettemy [1] sobre Koror, y de Glover [18] sobre Jarvis y Guam, y observar la inversión estacional de la variación magnética diaria de la declinación, D, obtenidas por Fukushima [2,3] para estudiar los efectos de las CAC en bajas latitudes magnéticas sobre Koror. Este estudio está basado en los valores medios horarios de $\mathrm{D}, \mathrm{H}$ y Z deducidos principalmente de los magnetógrafos normales, tabulados y publicados por Giesecke y otros [22], y se utiliza el método sugerido por Gettemy [1]. Este es un método que exhibe las variaciones magnéticas diarias usando mapas de contorno magnéticos. Este método puede dar una indicación más precisa de los cambios estacionales en la variación magnética diaria que otros métodos convencionales [1]. Para mostrar simultáneamente la variación magnética diaria y su cambio estacional en Huancayo, se elaboraron los mapas de contorno magnéticos mostrados en las figuras 3a, 3b, y 3c para DD, DH y DZ respectivamente. La hora del día es la abscisa, y el día del periodo AGI la ordenada. Para la elaboración de estos mapas de contorno se elaboraron tablas de desigualdades diarias medias para D, H y Z, utilizando el método propuesto por Chapman y Bartels [26]. Los resultados presentados en este trabajo están basados en todos los datos horarios disponibles del OMH durante los dos años 1957 y 1958, siendo aproximadamente 52,000 los datos usados y procesados. Un programa en Fortran [27] fue utilizado para el procesamiento de los datos del OMH durante el AGI y el AIEE, del OMA durante el AIEE y también para los otros observatorios ecuatoriales pero en una forma más simplificada. Este es un trabajo preparatorio para desarrollar técnicas de análisis que se utilizarán para tratar los datos que se hayan registrado durante el AIEE que abarca el periodo total de Enero de 1990 hasta Diciembre de 1994 y constituye un aporte al Programa Peruano de Geomagnetismo dirigido por el Instituto Geofísico del Perú (IGP). Los valores absolutos medios de los elementos del campo geomagnético mostrados en los mapas para el periodo 1957-1958 son como sigue:

\section{H : 28612 nT; D : 5²3' Oeste; Z : 989 nT}

Según Gettemy [1], los mapas de contorno magnético dan una buena información de la variación magnética diaria en un observatorio magnético ecuatorial. Se ha obtenido los mapas de contorno de la variación magnética diaria de las componentes $\mathrm{D}$, H y Z del campo geomagnético observadas en Huancayo sobre la región ecuatorial magnética del Perú para analizar los cambios estacionales durante el AGI. Mencionamos aquí una característica muy interesante de la variación magnética diaria Sq en el OMH. El OMH ha permanecido cerca al ecuador magnético desde que comenzó su operación en 1922. El valor medio mensual de Z fue de $984 \mathrm{nT}$ en enero de 1957 y $985 \mathrm{nT}$ en diciembre de 1958. Para el intervalo de cerca de 17:00 a cerca de 08:00 en tiempo local medio, las desviaciones horarias en nT de DH de su media diaria está confinada a valores entre - 25 nT y -50 nT; por lo tanto, la resolución utilizada para el gráfico no permite que ninguna línea de contorno pueda ser mostrado durante ese intervalo de tiempo. El tiempo del máximo de DH es siempre antes del medio día (aproximadamente 11:00 hs TL), más temprano que lo obtenido por Gettemy [1] en Koror. Este resultado confirma lo obtenido por Forbush y Casaverde [25]. La máxima desviación positiva de DH es alrededor de $170 \mathrm{nT}$ y ocurre durante los equinoccios de 1958. Huancayo se encuentra ligeramente al norte del ecuador magnético observado y en el hemisferio sur geográfico mientras que Koror se encuentra al sur del ecuador magnético observado y en el hemisferio norte geográfico. En el caso de Koror el máximo más pronunciado ocurre durante el equinoccio de verano del hemisferio norte (discrepando con los resultados de Gettemy [1]). Se puede concluir que ambos mapas de H tanto para Huancayo como para Koror son muy similares. La variación diaria DZ es siempre positiva por la mañana y negativa por la tarde durante los dos años. La variación DD presenta una inversión estacional, de la misma manera como se presenta en Koror. El mapa de contorno para DD muestra el cambio de patrón Sq, es decir, presenta una inversión estacional durante el verano del hemisferio sur geográfico, pero, no muy definida durante el invierno del hemisferio sur geográfico. Si se atribuye a la corriente eléctrica equivalente que cruza el ecuador del campo Sq a las CAC en la magnetósfera en bajas latitudes magnéticas, la inversión estacional de la variación diaria de DD en la región ecuatorial es atribuida a un par de sistemas de CAC en el hemisferio iluminado por el Sol cerca a los meridianos del amanecer y anochecer. De hecho, el eje central del EE evidentemente se ubica ligeramente al sur de Huancayo (cerca de Cañete y Cusco), como juzgamos del hecho que las desviaciones de DZ son negativos en el tiempo de DH máximo. Esto demuestra que efectivamente el OMH se encuentra, pues, en el hemisferio geográfico sur y al norte del ecuador magnético observado, es decir, se encuentra entre los dos ecuadores geográfico y magnético.

\section{Variación magnética diaria en Huancayo y Ancón durante el AIEE}

Durante el inicio del periodo referido como AIEE (Enero de 1990 hasta Diciembre de 1991) los valores máximos de DH en Huancayo se presentan en los equinoccios y aproximadamente a las 11 hs TL, con un máximo mayor de 150 nT durante Marzo de 1991 y un máximo menor de 147 nT durante Setiembre de 1990 y 125 nT en 1991. En cambio, en 1990 (durante el periodo Enero-Junio 1990) el valor máximo de DH ( 147 nT) se presenta en Febrero. Los valores mínimos de DH ocurren en el invierno del hemisferio sur. El mapa de contorno de DH en Huancayo durante el periodo 1990-1991 se muestra en la figura 4b. Los valores máximos de DH en Ancón también ocurren en los equinoccios durante el periodo de Julio de 1990 hasta Diciembre de 1991, con un máximo mayor en marzo de 1991 de 166 nT y a las 11 h TL, máximo menor de 149 nT en octubre de 1990 y a las 11 hs TL y otro máximo no muy bien 
definido que ocurre en setiembre de 1991 con una amplitud de $124 \mathrm{nT}$ a las 11 hs de TL. El OMA se encuentra aproximadamente a la misma latitud magnética observada o "dip" que el OMH y, como se esperaba, la variación magnética muestra el mismo comportamiento estacional para ambos periodos AGI y AIEE. El mapa de contorno para DH en Ancón se muestra en la figura $5 \mathrm{~b}$. Los mapas de contorno para DD y DZ en Huancayo y Ancón durante el AIEE se muestran en las Figuras 4a, 4c y 5a, 5c respectivamente, y son similares a los de Huancayo durante el AGI. Esta característica de la variación diaria DZ, que es independiente de la estación, parece incluir en una considerable extensión el efecto de las corrientes eléctricas en el mar que fluyen a lo largo de la costa del Perú, inducido por el desarrollo y decaimiento diario del intenso electrochorro sobre el Perú en el día. Las corrientes eléctricas inducidas fluyen hacia el norte en la mañana y hacia el sur en la tarde, produciendo un DZ positivo y negativo en el OMH y el OMA, respectivamente.

\section{Variación magnética diaria en días quietos en observatorios cerca al ecuador magnético durante AGI.}

Los mapas de contorno de la componente $\mathrm{H}$ del campo geomagnético para los observatorios ecuatoriales de Huancayo, Jarvis, Koror, Trivandrum y Addis Ababa (Ver tabla 1) para el año 1958 son mostrados en las figuras 6b, 7b, 8b, 9b y 10b respectivamente. En Huancayo y Jarvis los máximos menores ocurren en marzo (113 nT, 12 hs TL y 130 nT, 11 hs TL). En cambio, los máximos mayores ocurren en octubre (129 nT, 11hs TL) y Noviembre (135 nT, 11hs TL) respectivamente; ésto porque la intensidad de la radiacion solar es máxima en esas épocas. En Koror, el máximo menor ocurre en abril (144 nT, 13 hs TL) y el máximo mayor en octubre (157 nT, 12 hs TL). En Addis Ababa los máximos ocurren en abril y setiembre con una amplitud de aproximadamente 137 $\mathrm{nT}$ a las 11 hs TL. Finalmente, Trivandrum muestra sus máximos en abril y setiembre con los valores de $149 \mathrm{nT}$ y $137 \mathrm{nT}$ a las 11 hs de TL, es decir, con un máximo mayor en abril que en setiembre. Se puede concluir que todos ellos muestran sus máximos en la estación equinoccial y cerca al medio día. Además, Jarvis y Huancayo se encuentran en el hemisferio sur y se encuentran entre los ecuadores geográfico y magnético observados, mientras que Koror, Trivandrum y Addis Ababa se encuentran en el hemisferio norte y entre los ecuadores magnético observado y geográfico. Por lo tanto, Jarvis y Huancayo se encuentran al norte del EE y Koror, Trivandrum y Addis Ababa al sur del EE. Los mapas de contorno de la componente D del campo geomagnético para los observatorios ecuatoriales de Huancayo, Jarvis, Koror, Trivandrum y Addis Ababa para el año 1958 son mostrados en las figuras 6a, 7a, 8a, 9a y 10a respectivamente. Todos ellos muestran la inversión estacional aunque no es muy definido en Trivandrum. Los mapas de contorno de la componente $\mathrm{Z}$ del campo geomagnético para los observatorios ecuatoriales de Huancayo, Jarvis, Koror, Trivandrum y Addis Ababa para el año 1958 son mostrados en las figuras 6c, 7c, 8c, 9c y 10c respectivamente. Todos ellos muestran que DZ es positivo en la mañana hasta el medio dia, y negativo en la tarde, durante todo el año.

\section{Corrientes eléctricas alineadas con el campo geomagnético en bajas latitudes magnéticas en Perú}

Definimos aqui DX, DY y DZ como las desviaciones en los datos de X, Y, y Z de los cuatro valores promedios centrados en las 24 hs a $75^{\circ} \mathrm{WMT}$, suponiendo que los valores de medianoche puedan ser tomados como los valores de línea base para estudiar las variaciones geomagnéticas diarias que se originan de las corrientes eléctricas que fluyen en la ionósfera y magnetósfera. Un programa en Fortran [27] fue utilizado para el procesamiento de datos y así obtener los patrones Sq de los observatorios en Perú durante el AGI. Se ha realizado un nuevo análisis de las variaciones geomagnéticas diarias en tierra de la red de estaciones magnéticas peruanas durante el AGI. Esto permite inferir las CAC a bajas latitudes magnéticas. Estas corrientes eléctricas fluyen sobre el hemisferio de la Tierra iluminado por el Sol desde el hemisferio de invierno al hemisferio de verano en medianas latitudes. A bajas latitudes magnéticas cerca del ecuador, la dirección de las CAC es del hemisferio de verano al de invierno en las horas anteriores al mediodia y del hemisferio de invierno al de verano en las de la tarde. Las figuras 11 y 12 ilustran las direcciones de las corrientes eléctricas superiores para valores (DX, DY) y DZ (positivo verticalmente hacia abajo en el diagrama) para 1/2 (verano + invierno) y $1 / 2$ (verano-invierno). Si los patrones Sq fueran exactamente los mismos para ambas estaciones de verano a invierno, con su diferencia sólo en la magnitud, el patrón para 1/2(verano + invierno) podría ser el mismo como el de 1/2 (verano - invierno) excepto por la escala. La no similaridad de los patrones de corriente eléctricas Sq en las figuras 11 y 12 revela la diferencia entre las corrientes eléctricas Sq que se da entre el verano y el invierno. Según la figura 11, en Huancayo el sentido de las corrientes eléctricas es antihorario, de menor magnitud DZ que las demás; esto, quizás, por estar muy cerca del EE. En la figura 11 se nota un área DZ negativa centrada a las 9 hs TL sobre Talara, Chiclayo, Huancayo y Yauca asociado con una dirección de la corriente eléctrica superior horario y un área DZ negativo centrado a las 19 h TL sobre Talara, Chiclayo, Chimbote, Huancayo y Yauca asociado con una dirección de la corriente eléctrica superior en sentido horario. Esto puede ser una huella débil de las dos CAC en bajas latitudes magnéticas. En la figura 12 el área DZ es positivo en Talara, Chiclayo, Chimbote, y Huancayo, pero en Yauca es negativo cerca al medio dia. La enorme magnitud de DZ positivo en Yauca hace recordar la probable manifestación de la anomalía de la conductividad de los Andes. Se sugiere analizar los datos del AIEE de 1990-1994 con el mismo metodo para las diez estaciones peruanas instaladas.

\section{CONCLUSIONES}

La variación DD presenta una inversión estacional, bien definida durante el verano y no muy definida durante el invierno del hemisferio sur, como se muestra en las figuras 3b y 4b para el OMH y figura 5a para el OMA. Esta inversión está desplazada por 6 meses con respecto a Koror. Los mapas de contorno nos muestran que el máximo de la variación DH en OMH se encuentra antes del medio día durante el verano del hemisferio sur (cerca de las 11:00 hs TL) confirmando el resultado de Forbush y Casaverde [25]. Comparando figuras $3 \mathrm{~b}$ y $4 \mathrm{~b}$, con figuras 3a y 4a, obtenemos una impresión que el máximo de los rangos diarios DD en un año parecen tener lugar cuando los rangos diarios de DH son más pequeños. La variación DZ en el OMH y OMA es positivo en la mañana y negativo en la tarde independientemente de la estación durante los dos años. Esta característica de la variación diaria $\mathrm{DZ}$, que es independiente de la estación, parece incluir en una considerable extensión el efecto de las corrientes eléctricas que fluyen en el mar a lo largo de la costa del Perú, inducidas por el desarrollo y decaimiento diario del intenso EE hacia el este sobre el Perú durante el día. Los patrones de corrientes eléctricas superiores de Sq mostrados en las figuras 11 y 12 muestran las huellas débiles de las CAC en bajas latitudes magnéticas. La dirección de las corrientes eléctricas superiores en la figura 11 es con sentido horario en Talara, Chiclayo, Chimbote y Yauca, excepto en Huancayo a las 9 hs TL, y también a las 19 hs TL para las mismas 
estaciones. Se recomienda analizar con los mismos métodos los datos magnéticos recolectados por la red de estaciones magnéticas instaladas durante todo el periodo del AIEE. Se recomienda elaborar los mapas de contorno magnético de $\mathrm{D}, \mathrm{H}$ y Z del campo geomagnético de una estación magnética ecuatorial del Brasil durante el AIEE, para observar la variación estacional en la variación diaria y compararlos con los del OMH y OMA y por estar en el hemisferio sur y lejos del ecuador geográfico. De esta manera podemos estudiar la inversión estacional de DD para apoyar la hipótesis de las CAC en bajas latitudes magnéticas. La línea de contorno magnético cero se adelanta en el verano y se retraza en el invierno en el OMH y OMA. Esto ocurre antes del medio día. En Koror ocurre después del mediodía.

\section{AGRADECIMIENTOS}

Este trabajo de investigación fue financiado por el CONCYTEC y el IGP a través de una pasantía de investigación otorgada a uno de los autores (J.R.A.). Agradecemos al personal administrativo y docente de la Facultad de Ciencias Físicas de la Universidad Nacional Mayor de San Marcos por habernos facilitado el equipo y material necesarios para el procesamiento y análisis de los datos. Agradecemos también al Dr. Mutsumi Ishitsuka quien, como Director del Observatorio Magnético de Ancón (OMA), proporcionó los datos magnéticos de los observatorios de Huancayo (OMH) y de Ancón durante el AIEE y el AGI. Y, finalmente, a los miembros del Proyecto Especial de Geomagnetismo del Observatorio de Ancón por su valioso apoyo técnico.

\section{REFERENCIAS}

[1] J.W.Gettemy. Magnetic daily variation at Koror, J. Geohys. Res., 67: 1885?1888. (1962)

[2] N. Fukushima. Field-Aligned Currents in the Magnetosphere, Geof. Int. 30/4: 241?248 (1991)

[3] N. Fukushima. Transequatorial field-aligned currents at low latitudes and their possible connection with the equatorial electrojet, Revista Brasileira de Geofísica, 11 (3, especial): 291-301 (1993)

[4] D. van Sabben. North - South asymmetry of Sq. J. Atmos. Terr. Phys., 26: 1187-1195 (1964)

[5] D. van Sabben. Magnetosheric currents, associated with the N-S asymmetry of Sq. J.A.T.P., 28: $965-981$ (1966)

[6] A. J. Zmuda, J. H. Martin y F.T. Heuring. Transverse magnetic disturbances at 1100 kilometers in the auroral region. J. Geophys. Res., 71: 5033-5046. (1966)

[7] D. van Sabben. Solstitial Sq-currents through the magnetosphere. J. Atmos. Terr. Phys., 32: 1331-13336. (1970)

[8] K. Yanagihara. Geomagnetic variations near focus of Sq current vortex. Memoirs Kakioka Mag. Obs., 14: 1-8 (1971)

[9] H. Maeda. Field-Aligned current induced by asymmetric dynamo action in the ionosphere. J. A. T. P., 36: 1395-1401 (1974)

[10] N. Fukushima. Electric potential difference between conjugate points in middle latitudes caused by asymmetric dynamo in the ionosphere. J. Geomag. Geoelectr., 31: 401-409 (1979)

[11] C.U. Wagner, K. Schafer and V. M. Mishin. A model of field-aligned currents inside the undisturbed plasmasphere. Gerlands Beitr. Geophys., 92: 353-360 (1983)

[12] N. Fukushima. Comunicación personal (1994)

[13] N. Fukushima. Equivalence in ground magnetic effect of Chapman-Vestine's and Birkeland-Alfven's electric currents-systems for polar magnetic storms. Rept. Ionos. Space. Res. Japan, 23: 219-227 (1969)

[14] N. Fukushima. Electric currents systems for polar magnetic storms and their magnetic effect below and above the ionosphere, Radio Sci., 6: 269-275 (1971)

[15] N. Fukushima. Generalized theorem for no ground magnetic effect of vertical currents connected with Pedersen currents in the uniform-conductivity ionosphere. Rept. Ionos. Space. Res., Japan,30: 401-409 (1976)

[16] D. van Sabben. The computation of magnetospheric currents, caused by dynamo action in the ionosphere. J. Atmos. Terr. Phys., 31: 469-474 (1969)

[17] C.A. Onwumechilli and N.S. Alexander. Variations in the geomagnetic field at Ibadan, Nigeria-I. Solar variation, J. Atmos. Terr. Phys., 16: 106 - 114 (1959)

[18] F.N. Glover. Recent magnetic observations in the Philippines, J. Geophys. Res., 68: 2385 - 2394 (1963)

[19] V.R. Hutton. Sq currents in the American equatorial zone during the I.G.Y., I. Seasonal effects. J. Atmos. Terr. Phys., 29: 1411$1428(1967 \mathrm{a})$

[20] V.R. Hutton. Sq currents in the American equatorial zone during the I.G.Y., II. Day-to-day variability. J. Atmos. Terr. Phys., 29: 1429-1442 (1967b)

[21] K. Maeda y H. Murata. Ionospheric dynamo theory with consideration for magnetospheric current along the geomagnetic lines of force, Rept. Ionos. Space. Res., Japan, 19: 272-285 (1965)

[22] A.A. Giesecke, M. Casaverde y P.G. Ledig. Vol. X-D, tomos I al VII, 1948 - 1961, I.G.P. (1962)

[23] M. Ishitsuka. comunicacion personal (1993)

[24] Price y Stone. The quiet-day magnetic variations during the IGY, Annals of the IGY, 35: 63-269 (1964)

[25] S.E. Forbush y M. Casaverde. Equatorial electrojet in Perú, Carnegie Inst. Wash. Publ. 620 (1961)

[26] S. Chapman y J. Bartels. Geomagnetism, Oxford Press, London, 2 vol. (1940)

[27] A.J. Rojas. Corrientes Alineadas con el Campo Geomagnético en Bajas Latitudes Magneticas, Tesis de Maestria en Física, Facultad de Ciencias Físicas, Universidad Nacional Mayor de San Marcos (1994)

[28] K. Burrows. The day-to-day variability of the equatorial electrojet in Perú. J. Geophys. Res., 75/7: 1319-1323 (1970)

[29] V.M. Mishin. Quiet geomagnetic variations and currents in the magnetosphere, "Nauka", Sib. Otdel., Novosibirsk (1976).

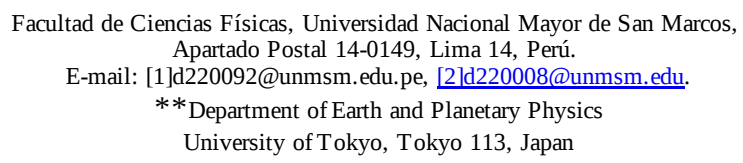


